\title{
Radiative recombination in initial and electron-irradiated GaP crystals
}

\author{
O. Hontaruk ${ }^{1}$, O. Konoreva ${ }^{1}$, P. Litovchenko ${ }^{1}$, V. Manzhara ${ }^{1}$, V. Opilat ${ }^{2}$, M. Pinkovska ${ }^{1}$, V. Tartachnyk ${ }^{1}$ \\ ${ }^{1}$ Institute for Nuclear Research, NAS of Ukraine, 47, prospect Nauky, 03028 Kyiv, Ukraine \\ ${ }^{2}$ M. Drahomanov National Pedagogical University, 9, Pirohova str., 01601 Kyiv, Ukraine \\ Corresponding author phone: (+044)-525-39-97; e-mail: okskon@meta.ua
}

\begin{abstract}
Photoluminescence of GaP crystals irradiated by $1 \mathrm{MeV}$ electrons was studied at $4.2 \mathrm{~K}$. Samples were prepared using various technologies and doped by $\mathrm{Te}, \mathrm{Zn}, \mathrm{Mg}$ and N. Emission spectra were analyzed as dependent on the impurity content. Found was the electron irradiation influence on the luminescence intensity and its mechanism. Radiative recombination intensity was shown to recover efficiently within the temperature range $200-600{ }^{\circ} \mathrm{C}$, and the main annealing stage being at $200-400{ }^{\circ} \mathrm{C}$.
\end{abstract}

Keywords: GaP, photoluminescence, recombination, degradation.

Manuscript received 01.10.09; accepted for publication 22.10.09; published online 04.12.09.

\section{Introduction}

The researches of structural defects in $\mathrm{GaP}$ monocrystals, which started at the end of 60 -ths, are still far from the completion. The number of published works concerning these problems increases rapidly; many of them deal with radiation-induced lattice defects [1-9].

In recent years, the interest to these materials reinstates because of the increase in the field of $\mathrm{GaP}$ oscillators (emitters) application. Light emitting structures, used earlier only as active elements in optoelectronics industry, has become high sensitive thermometers, detectors, high temperature diodes [7-9].

From the viewpoint of examination of variety radiative recombination mechanisms, one can consider $\mathrm{GaP}$ crystals to be the model ones. Due to the great oscillator strength and the low depth of the trap, namely isovalent nitrogen impurity, the emitting source with the quantum energy close to the forbidden gap might be obtained. The green band of the visible spectrum responds to such transition. In homogeneous crystals without dislocations, the band of a free exciton with series of the phonon reiteration can also exist. Intentionally doped crystals show a donor-acceptor pair emission [10-11].

So, a luminescence spectrum of high pure crystals is known enough [12-14]; but the origin of main bands of $\mathrm{GaP}$ and materials on its base, exploitable in electronic industry, are not yet resolved and need subsequent study.

\section{Experiment}

Optical characteristics are known to be highly sensitive to the lattice defects, that is why we used them in our investigations. Samples were made by different technologies: by Czochralski method, from solutionmelting and epitaxy. Te-, Zn-, Mg- and N-doped crystals were irradiated at electron accelerator ELT-1.5 with electrons at the temperatures not higher than the room ones. The electron energy was $1 \mathrm{MeV}$. Photoluminescence of the samples was measured at $4.2 \mathrm{~K}$. Excitation was carried out by a quartz lamp through the water filter. Radiation induced suppression of luminescence and the influence of thermal annealing were studied. The origin of emission mechanisms is discussed.

\section{Results and discussion}

Electroluminescence characteristics of various samples at $4.2 \mathrm{~K}$ are shown below.

Fig. 1 presents spectra of undoped $n$-type $\mathrm{CZ} \mathrm{GaP}$ with three broad structureless bands at $2.174,2.224$ and $2.28 \mathrm{eV}$.

Considering the value of GaP forbidden gap energy $2.34 \mathrm{eV}(4.2 \mathrm{~K})$, one can evaluate the depth of the level responding to the emitting transition $2.28 \mathrm{eV}$. A donor with the energy $E_{d}=0.06 \mathrm{eV}$ might be such a trap. This value is close to the ionizing energy of uncontrolled $\mathrm{Sn}$ impurity $(0.058 \mathrm{eV})$ [15]. As its concentration is not high, the band intensity is low. 




Fig. 1. Luminescence spectrum of undoped $n$-type $\mathrm{CZ} \mathrm{GaP}$ at $4.2 \mathrm{~K}$.

The origin of the transition at $h v_{2}=2.224 \mathrm{eV}$ is probably related with donor-acceptor transitions. If the emitted quantum energy is

$$
h v=E_{g}-\left(E_{d}+E_{a}\right)+\frac{e^{2}}{\varepsilon r}-\Delta E,
$$

with $E_{g}$ is the forbidden band width; $E_{d}, E_{a}$ - energies of donor and acceptor levels, accordingly; $r$ - distance between atoms; $\varepsilon$ - dielectric permittivity;

$$
\Delta E=\frac{e^{2} b^{5}}{\varepsilon r^{6}}, b=(6.5)^{1 / 5} a_{0},
$$

where $a_{0}$ is the Bohr radius of carriers with a higher bond energy [1].

Then, in the case $r \rightarrow \infty$

$$
h v=E_{g}-\left(E_{d}+E_{a}\right) \text {. }
$$

When undoped $\mathrm{GaP}$ possesses uncontrolled $\mathrm{Zn}$ impurity $\left(E_{\mathrm{Zn}}=0.064 \mathrm{eV}\right)$, the intensity maximum of far laying pairs is $2.218 \mathrm{eV}$; this value is close to $h v_{2}$. The difference between experimental and calculated data is explained by the band maximum dependence $h v_{2}=2.224 \mathrm{eV}$ on the concentration of donor-acceptor pairs.

So, we consider the second band is the superposition of discrete emitting lines of donoracceptor $\mathrm{Sn}-\mathrm{Zn}$ pairs separated by long distances.

The low-energy maximum $h v=2.17 \mathrm{eV}$ is the most probably formed by phonon reiteration of the main band maximum. They differ by the distance that equals to the GaP photon energy (45 meV).

The absence of the fine structure of pair emitting even at $4.2 \mathrm{~K}$ testifies about a high content of defects in undoped $\mathrm{GaP}$.

In Mg-doped CaP (Fig. 2) an additional emitting band $h v_{2}=2.296 \mathrm{eV}$ appears, which is identified as the transition onto the $0.044 \mathrm{eV}$ level located near the valence band top. This value is close to $\mathrm{Mg}$ acceptor impurity energy $(0.052 \mathrm{eV})[15]$.

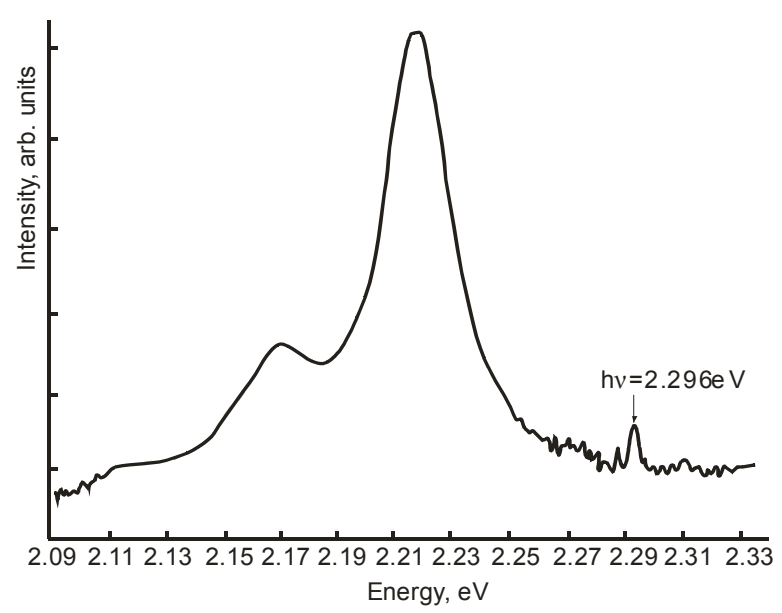

Fig. 2. Luminescence spectrum of $\mathrm{Mg}$ doped $\mathrm{GaP}$ at $4.2 \mathrm{~K}$.

In $\mathrm{Zn}$-doped $p$-type $\mathrm{GaP}$, in addition to the main band maximum $2.218 \mathrm{eV}$ the band $h v=2.280 \mathrm{eV}$ appears, which is related with the emitting transition onto the acceptor level $0.060 \mathrm{eV}$. The latter is probably related to $\mathrm{Zn}$, as $E_{\mathrm{Zn}}$ value coincides with known lines within the range $0.064-0.617 \mathrm{eV}[10,15]$.

Epitaxial $\mathrm{GaP}$ of $n$-type displaces broad structureless band with the maximum close to $2.153 \mathrm{eV}$ (Fig. 3). This value coincides with the envelope of peak series for the phonon reiteration of the $\mathrm{NN}$ lines found in [16] by studying the GaP:N diodes. So, green emitting band in epitaxial GaP is also caused by $\mathrm{N}$ impurity and has excitonic nature.

In GaP crystal grown from solution-melting, a fine structure appears in the emission spectrum (Fig. 4).

The origin of main lines was studied in the classical Gershenson research [17] as well as in other works [1819]. As it was already shown, different A-, B-, C-lines are splitted in magnetic field, which is indicative of the

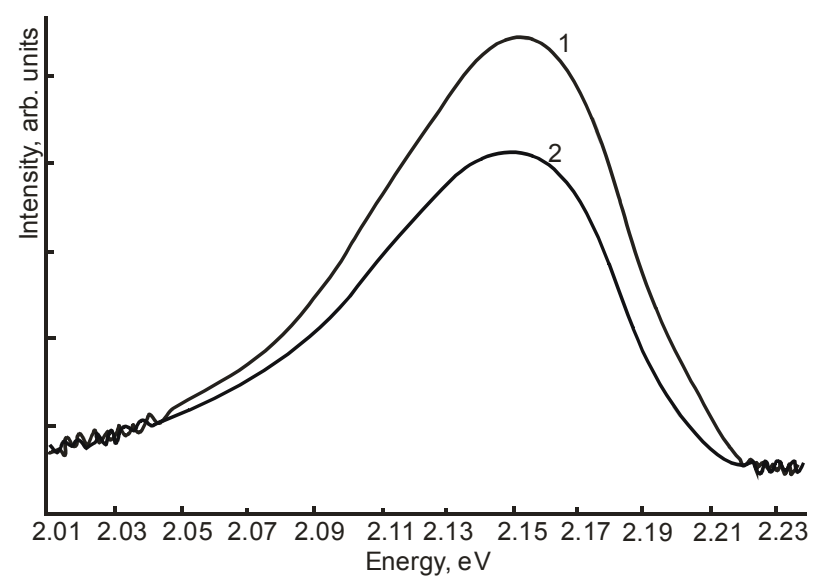

Fig. 3. Luminescence spectra of $\mathrm{GaP}$ made by the liquid epitaxy method at $4.2 \mathrm{~K}: 1-$ initial, 2 - after electron irradiation $\left(E_{e}=1 \mathrm{MeV}, \Phi=10^{14} \mathrm{~cm}^{-2}\right)$. 
exciton emission origin [17]. The first and the second lines are doubling components with the distance in maxima $10^{-4} \mathrm{eV}$. A- and B-lines are related with direct excitons bounded on the ionized center, while C-line is the result of annihilation with the indirect exciton located on a neutral donor.

Emission spectra of two GaP solution-melting grown samples with the purity higher than in the previous case are given in Figs 5a,b (a - the most pure crystal, $b$ - medium, Fig. 4 - the worst). The maximum of the narrow line with the highest intensity (D-line) at $2.337 \mathrm{eV}$ evidently responds to the free exciton emission. The nearest adjoining line $2.331 \mathrm{eV}$ is $6 \mathrm{meV}$ lower than D-line, and one could identify it with the DTA phonon reiteration $(\mathrm{TA}=8.2 \mathrm{meV})$. But in the medium purity crystal, it is the main line in the spectrum (Fig. 5b), so we can consider its origin as caused by the recombination of exciton located on uncontrolled impurity.

Izoelectron $\mathrm{N}$ impurity in $\mathrm{GaP}$ is highly electronegative. While joining the electron, nitrogen becomes the charge center and attracts a hole, associated with G-maximum of the valence band.

Then indirect exciton is formed with the radius stemming from the equation

$a_{\mathrm{ex}}=\varepsilon\left(\frac{m_{r}}{m_{0}}\right)^{-1} a_{B}$,

where $m_{0}$ is the free electron mass, $m_{r}-$ specific mass, $a_{B}=0.53 \AA$ ( $a_{B}-$ Bohr radius).

If $\varepsilon=11.11$ in $\mathrm{GaP}$, then $a_{\mathrm{ex}}=17 \AA \AA$ and responds to $m_{r} / m_{0}=0.346$, which means that we deal with the exciton of large radius, namely the Wannier exciton.

Its bond energy on an isolated atom is approximately equal to $21 \mathrm{meV}$ [11], and emission energy is $2.319 \mathrm{eV}$.

In the spectra of both samples, the line of excitons bounded to a nitrogen atom is denoted as N-line. The rest lines in the fine structure are the phonon reiteration of the free exciton as well as the exciton bounded to uncontrolled unknown impurity and nitrogen.

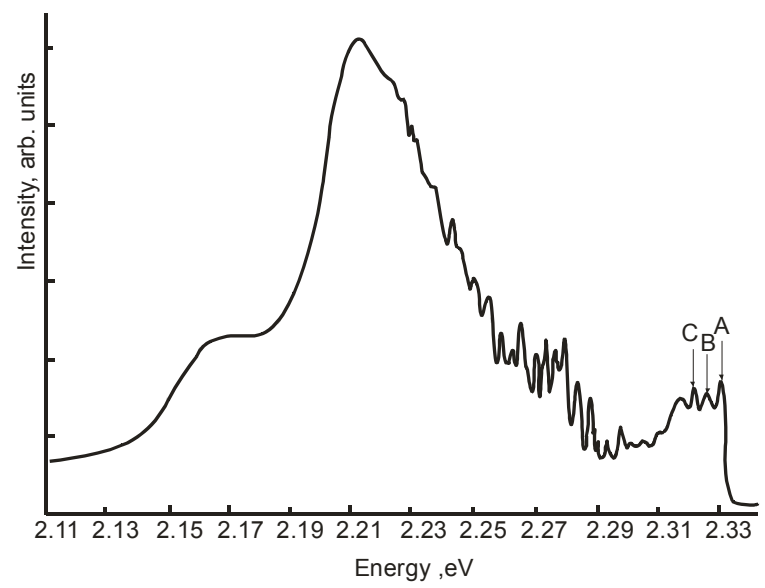

Fig. 4. Luminescence spectrum of $\mathrm{GaP}$ made by the solutionmelting method at $4.2 \mathrm{~K}$.
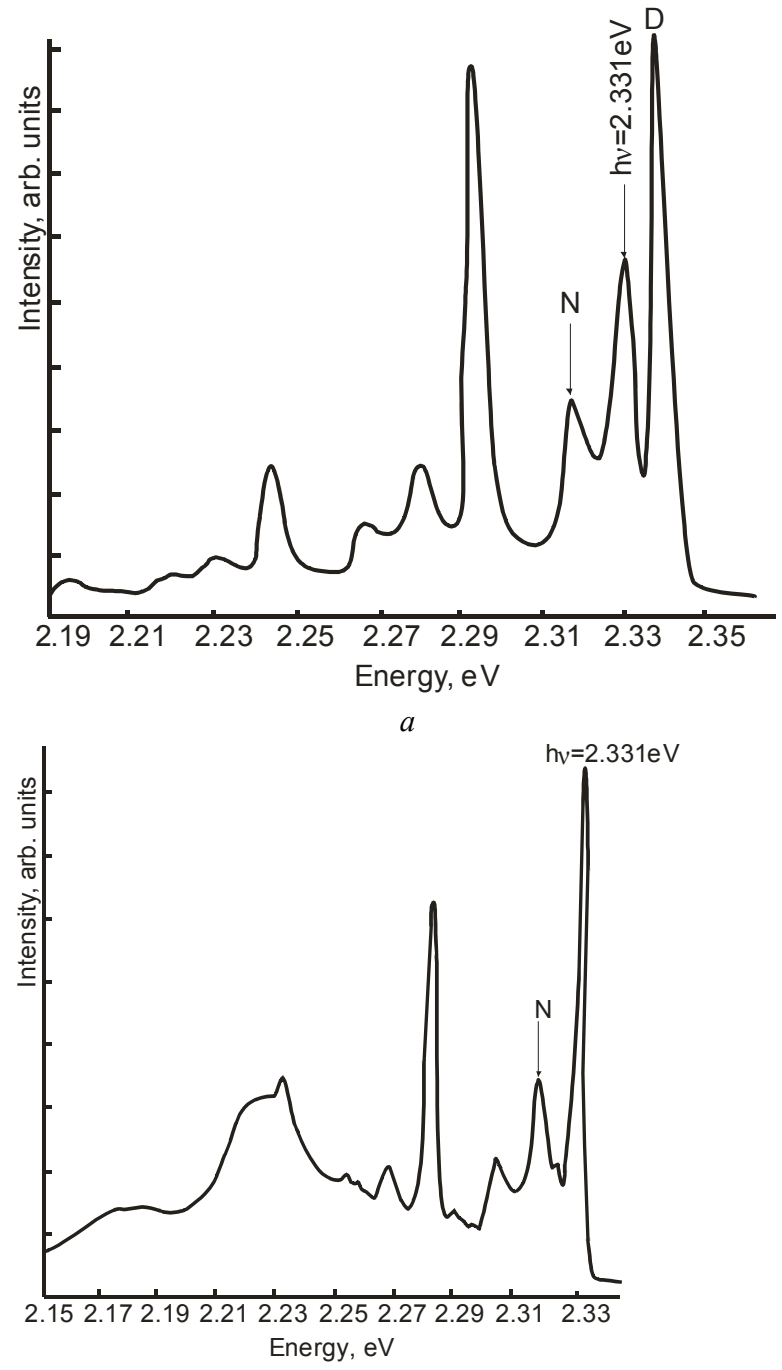

$b$

Fig. 5. Luminescence spectra at $4.2 \mathrm{~K}$ of $\mathrm{GaP}$ made by liquid epitaxy method with different crystal purity: $a$ - the higher purity level than $b$.

Irradiation of these samples by $1 \mathrm{MeV}$ electrons with the dose $10^{14} \mathrm{~cm}^{-2}$ leads to the intensity decrease in all the lines: by 2.82 times for free excitons and 3.11, 5.46 and 3.09 times for A-, B- and C-lines, accordingly. The C-line appears to be the most sensitive to radiation and has the lower temperature stability, as its origin is related with forbidden direct transitions [17]. It is probably the result of annihilation of excitons weekly bounded to the impurity center. C-line, in contrast with two others, is a result of its localization on neutral atom. The authors [17] proposed $\mathrm{Si}$ as playing the role of this center. But considering the close energy position of C-line $(2.310 \mathrm{eV})$ and nitrogen $(2.318 \mathrm{eV})$ and the same emission mechanism as the result of annihilation of indirect bond exciton, we concluded that N-line of our GaP spectra and C-line in [17] are identical. Nearly the same radiation hardness of this line with free and bond exciton lines is an additional confirmation of its exciton origin. 
The spectrum of the epitaxially grown GaP irradiated with electrons (curve 2, electron fluence $10^{14} \mathrm{~cm}^{-2}$ ) is given in Fig. 3 together with the initial one (curve 1). In comparison with the crystal prepared using the solution-melting, the decrease of the emission intensity after electron irradiation is much less (by 1.3 times) despite the same recombination mechanism. It is related with different concentrations of initial structural defects in both technologies. The velocity of incorporation of radiation defects into semiconductor depends on the Fermi level position inside the forbidden gap $E_{g}$ and decreases, while the Fermi level moves to the middle of $E_{g}$. In the compensated material, this velocity is less, so the crystal prepared from solution-melting is more perfect than GaP epitaxial films.

The position of main emission maximum also testifies to this assumption. In the irradiated GaP sample, the intensity maximum shifts to the lower quantum energy by $0.005 \mathrm{eV}$, while in the epitaxial material - this shift is $0.034 \mathrm{eV}$ from the emission maximum in the solution-melting grown crystal. Fields of structural defects influence on far NN-pairs, bond energy in which is less than that in the nearest pairs. So, the radiation sensitivity of maximum band intensity is the greatest (increases 10 times at the fluence $10^{14} \mathrm{~cm}^{-2}$ ).

Initial Mg-doped $\mathrm{GaP}$ is sufficiently defective, so even for the greater fluence $\left(5 \times 10^{14} \mathrm{~cm}^{-2}\right)$ photoluminescence intensity drops only by 2.36 times.

$\mathrm{CZ}$ grown undoped $\mathrm{GaP}$ has the same radiation tolerance, as its low quantum yield is caused by the high dislocation density.

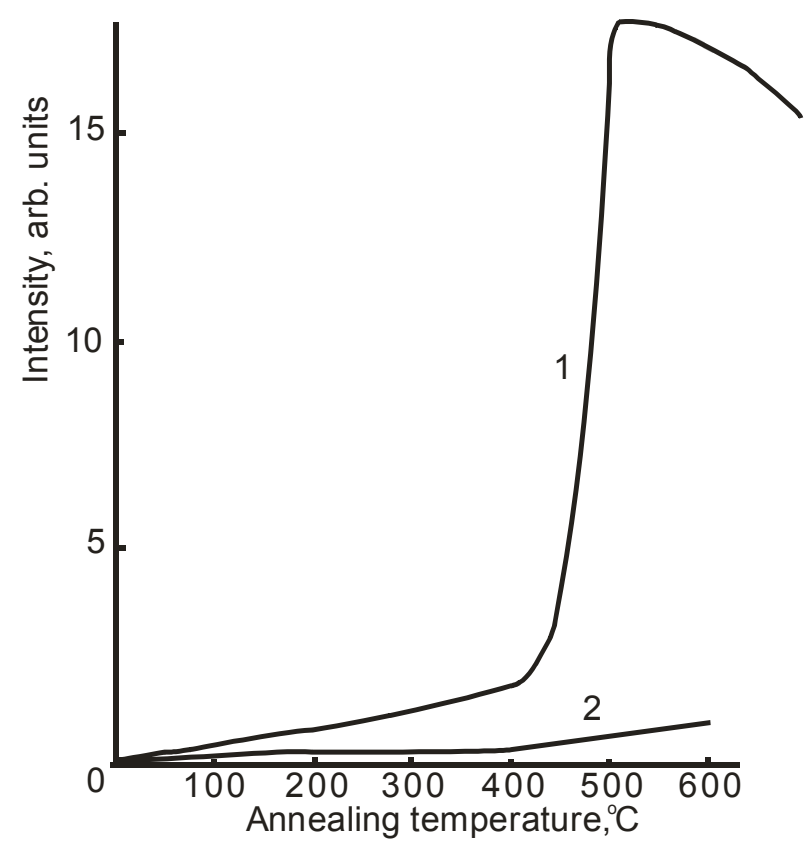

Fig. 6. Restoring the emission characteristics in $\mathrm{GaP}$ previously irradiated by $1 \mathrm{MeV}$ electrons $\left.\left(\Phi=10^{14} \mathrm{~cm}^{-2}\right): 1\right)$ samples grown by solution-melting; 2) GaP doped by $\mathrm{Mg}$.
After irradiation, GaP crystals were annealed. In $\mathrm{GaP}$ samples grown by solution-melting, restoring the emission characteristics in the process of heating begins after $100{ }^{\circ} \mathrm{C}$. The curve main band maximum increases slowly to $400{ }^{\circ} \mathrm{C}$, after that sharp restoring stage occurs within the $400-500{ }^{\circ} \mathrm{C}$ temperature range. Heating over $600{ }^{\circ} \mathrm{C}$ causes opposite processes - the luminescence intensity begins to decrease (Fig. 6).

Mg-doped GaP crystal anneals slowly; the intensity increase even at $600{ }^{\circ} \mathrm{C}$ is nearly an order less than for the samples grown by solution-melting. The same behavior is typical for $\mathrm{CZ}$ grown $\mathrm{GaP}$.

Concerning the fine structure of $\mathrm{GaP}$ grown by solution-melting, its restoring after irradiation begins at $200{ }^{\circ} \mathrm{C}$ : in the annealed samples the free exciton line appears at $400{ }^{\circ} \mathrm{C}$ again together with LO-background reiteration (Fig. 7). Nearby there is the series of close laying lines with the $2.5 \mathrm{eV}$ mean distance between them. The most intensive lines are the background reiteration of a free exciton line. The weakest ones between background replica might respond to exciton annihilation from the excited state with participation of phonons. When considering the transition from the nearest state $n=2$ into $n=3$ and use experimental value $E_{\mathrm{ex}}=11 \mathrm{meV}$, then following relation may be written $E_{\mathrm{ex}}=-\frac{m_{e}^{*} q^{4}}{2 h^{2} \varepsilon^{2}} \frac{1}{n^{2}}$

where $q$ - electron charge, $h$ - Planck's constant, $n-$ quantum number $(n \geq 1)$.

The difference between two exciton excited states $\Delta_{1}=1.53 \mathrm{meV}$. In the mentioned region of the fine structure, the mean distance between lines is equal $\Delta_{2}=1.53 \mathrm{meV}$, which confirms our conclusion.

When annealing temperature increases to $500{ }^{\circ} \mathrm{C}$, the intensity of all the lines increases with some step in the longwave side (M-line in Fig. 8). The further heating at $700{ }^{\circ} \mathrm{C}$ causes degradation of emission in all parts of the spectrum due to thermal effects [24].

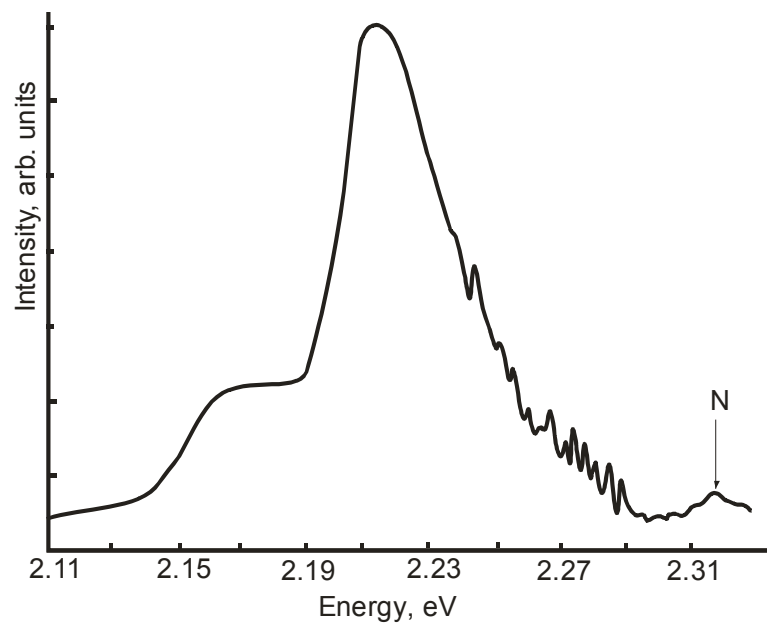

Fig. 7. Luminescence spectrum of $\mathrm{GaP}$ made by the solutionmelting method at $4.2 \mathrm{~K}$. Irradiated samples were annealed at $400{ }^{\circ} \mathrm{C}$. 


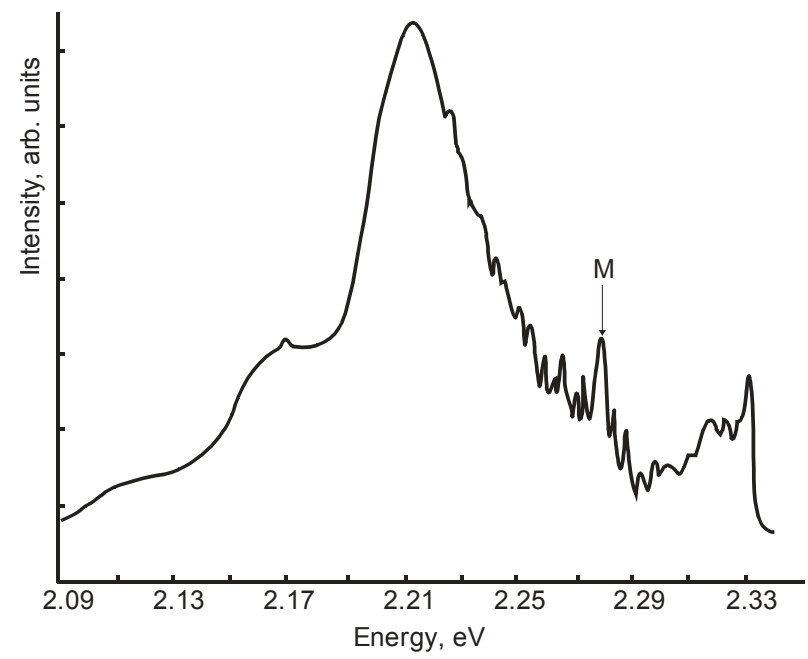

Fig. 8. Luminescence spectrum of GaP made by the solutionmelting method at $4.2 \mathrm{~K}$. Irradiated samples were annealed at $500{ }^{\circ} \mathrm{C}$.

$\mathrm{GaP}$ is a semiconductor widely used in optoelectronics, so its radiation hardness is determined as emitting capacity and optical absorption. It is clear from experiments that degradation of emission begins at the fluence $\Phi=10^{14} \mathrm{~cm}^{-2}$ : at $\Phi=5 \times 10^{14} \mathrm{~cm}^{-2}$ exciton spectra are fully destroyed, while in near-edge optical absorption this degradation occurs at $\Phi \approx 10^{14} \mathrm{~cm}^{-2}$ [14]. That is, the exciton spectrum component is much more sensitive to ionizing radiation than the optical one in the region of fundamental transitions. This difference is caused by the fact that exciton with low dissociation energy (dozen of $\mathrm{meV}$ ) doesn't withstand to radiation defect local fields even at long distances.

If to compare luminescence spectra of two GaP samples of different purity, one can see that the free exciton lines and background replica in luminescence spectra of more pure crystal are more intensive (Fig. 5a,b). In the worse cleaned crystal, the exciton concentration is low, and spectral lines are of low resolution (Fig. 3), because impurities distort the periodical field profile.

The same influence on exciton emission is inherent to thermal defects appearing after high temperature heating $\left(>600{ }^{\circ} \mathrm{C}\right)$. In irradiated samples, the effect is stronger due to formation of radiation defect-impurity complexes [24].

The near-edge absorption changes are the result of appearance of the density-of-states tails, which distort band edges; the effect is significant at high concentrations of structural defects.

\section{Conclusions}

Photoluminescence spectra of GaP crystals undoped and doped by Te, $\mathrm{Zn}, \mathrm{Mg}$ and $\mathrm{N}$ were studied at $4.2 \mathrm{~K}$.
The samples were prepared using different technologies and emission spectra were analyzed as dependent on the impurity content. In undoped $n$-type $\mathrm{GaP}$, two broad bands at $h v_{1}=2.28 \mathrm{eV}$ and $h v_{2}=2.224 \mathrm{eV}$ are present. The first band is related with an uncontrolled Sn impurity; the second one is the result of superposition of lines corresponding to donoracceptor transitions between distant $\mathrm{Sn}-\mathrm{Zn}$ pairs. The low-energy maximum $h v=2.17 \mathrm{eV}$ is formed by TOphonon reiteration of the main band maximum. The absence of the fine structure in the spectra indicates a high defect content in undoped $\mathrm{GaP}$.

The band $h v=2.296 \mathrm{eV}$ in Mg-doped $\mathrm{GaP}$ has been identified as the electron transition on the $0.044 \mathrm{eV}$ level near the valence band top and is related with $\mathrm{Mg}$ impurity.

In $\mathrm{Zn}$-doped $p$-type $\mathrm{GaP}$, the main $\mathrm{Zn}$ impurity $\left(E_{v}=0.064-0.062 \mathrm{eV}\right)$ responds to the $h v=2.280 \mathrm{eV}$ band maximum emission.

Green emission band in epitaxial $\mathrm{GaP}$ appears due to the far lying NN-pair emission.

In emission spectra of $\mathrm{GaP}$ crystal grown by solution-melting, the fine structure is observed: it consists of lines of free excitons and excitons bounded on a nitrogen atom and also its phonon reiteration.

Electron irradiation causes decrease of the photoluminescence intensity. The lowest radiation hardness is characteristic to the far lying NN-pair emission (its intensity drops by the factor 10 for the electron fluence $10^{14} \mathrm{~cm}^{-2}$ ).

Intensity restoring in the process of isochronous heating occurs within $100-600^{\circ} \mathrm{C}$ range, and in the highly pure crystal grown by solution-melting, a sharp stage of the luminescence intensity increase is observed at $400-600{ }^{\circ} \mathrm{C}$, followed at higher temperatures by degradation of emission due to creation of hightemperature thermal defects.

\section{References}

1. T. Endo, T. Nishimura, K. Nakakuki, M. Kitamura, K. Sugijama, DLTS study for energy-broadening of the defect level on introducing radiation damage in GaP // Jpn. J. Appl. Phys. 27(11), p. 2107-2112 (1988).

2. T. Endo, E. Uchida, Y. Hirosaki, K. Sugijama, Deep levels in non-doped and donor-doped GaP // Jpn. J. Appl. Phys. 27(1), p. 153-154 (1988).

3. T. Endo, Y. Hirosaki, E. Uchida, H. Miyake, K. Sugijama, Deep levels in electron-irradiated $\mathrm{GaP}$ at $10 \mathrm{MeV} / /$ Jpn. J. Appl. Phys. 28(10), p. 1864-1870 (1989).

4. M.A. Zaidi, M. Zazoui, J.C. Bourgoin, Defects in electron irradiated n-type GaP // J. Appl. Phys. 74(8), p. 4948-4952 (1993).

5. K. Kuriuama, Y. Miyamoto, Redshift of the longitudinal optical phonon in neutron irradiated GaP // J. Appl. Phys. 85(7), p. 3499-3502 (1999). 
6. A.V. Gommonai, D.B. Goyer, O.O. Goushchaa, Yu.M. Azhniuk, I.G. Megela, M. Kranjcec, Radiative recombination in electron-irradiated $\mathrm{GaP}$ crystals // J. Optoelectron. and Adv. Mater. 5(3), p. 641-646 (2003).

7. M. Zafar Iqbal, N. Baber, M. Arhad, N. Zafar, Sensitive thermometry using capacitance variation of GaP LEDs // Solid-State Electronics 30(6), p. 639-641 (1987).

8. I. Prochazka, K. Hamal, B. Sopko, J. Blazey, D. Chren, Photon counting detector with picosecond timing for $\mathrm{X}$ to visible range on the basis of GaP // Nucl. Inst. and Meth. Phys. Res. A 568, p. 437-439 (2006).

9. M.M. Sobolev, V.G. Nikitin, High-temperature diode formed by epitaxial GaP layers // Tech. Phys. Lett. 24(5), p. 329-331 (1998).

10. A. Berg, P. Din, Light Emitting Diodes. Mir, Moscow, 1979 (in Russian).

11. K. Zdansky, J. Zavadil, D. Nohavika, S. Kugler, Degradation of commercial high-brightness GaP:N green light emitting diodes // J. Appl. Phys. 82(12), p. 7678-7684 (1998).

12. M.I. Natham, G. Burns // J. Phys. Rev. 12(9), p. 125 (1963).

13. F. Williams // Phys. status solidi 25, p. 493 (1968).

14. D.I. Dean, Izoelectronic traps in semiconductors (experimental) // J. Lumin.7, p. 51 (1973).

15. V.M. Andreev, L.M. Dolginov, D.N. Tretyakov, Liquid Epitaxy in Technology of Semiconductor Devices. Sovetskoe radio, Moscow, 1975 (in Russian).
16. G.A. Sukach, Radiation-induced transformation of radiative exciton complexes bound to nitrogen in GaP:N green light-emitting structures // J. Lumin. 85, p. 121-128 (1999).

17. D.G. Thomas, M.E. Gershenson, Bound exitons in GaP // Phys. Rev. 131(5), p. 2397-2404 (1964).

18. M. Gal, Temperature modulated photoluminescence of GaP:N // Phys. Rev. B 18(2), p. 803-808 (1978).

19. W. Lancerns, A. Wiersma, Optical dephasing and exciton transfer of an impurity bound in semiconductors. Proton-echo experiment on GaP:N // Phys. Rev. B 32(12), p. 8108-8115 (1985).

20. G.A. Herrmannsfeldt, Yia-Chung Chang, Highpressure studies of luminescence from $\mathrm{GaP}$ and GaP:N diodes // Phys. Rev. B 34(8), pp.5373-5376 (1986).

21. V.S. Vavilov, Effect of Radiations on Semiconductors. Mir, Moscow, 1963 (in Russian).

22. Q. Hong, K. Wu, X. Zhang, Study on the luminescence of GaP:N under selective excitation of exciton bound to $\mathrm{NN}_{1}$ centers // J. Lumin. 40\&41, p. 487-488 (1988).

23. E.Yu. Brailovskii, N.I. Ostashko, V.P. Tartachnyk, V.I. Shakhovtsov, Influence of point radiation defects on near-edge optical absorption of $\mathrm{GaP}$ crystals // Ukrainskii Fizicheskii Zhurnal 26(6), p. 973-977 (1981), in Russian.

24. V.V. Volkov, V.Ja. Opilat, V.P. Tartachnyk, I.I. Tychyna, Deep levels in the initial and exposed to electrons beams gallium phosphide // Vysokochistye veschestva 2, p. 60-63 (1989), in Russian. 